\title{
Age at Menopause and Risk of Developing Endometrial Cancer: A Meta-Analysis
}

\author{
Yanjun Wu $(\mathbb{D}$, Wenjun Sun $(\mathbb{D}$, Hui Liu, and Dongfeng Zhang $\mathbb{D}$ \\ Department of Epidemiology and Health Statistics, The College of Public Health of Qingdao University, Qingdao, \\ Shandong Province, China \\ Correspondence should be addressed to Dongfeng Zhang; zhangdf1961@126.com
}

Received 22 October 2018; Revised 16 April 2019; Accepted 8 May 2019; Published 29 May 2019

Academic Editor: Joaquim Radua

Copyright (C) 2019 Yanjun Wu et al. This is an open access article distributed under the Creative Commons Attribution License, which permits unrestricted use, distribution, and reproduction in any medium, provided the original work is properly cited.

Object. The association of age at menopause with endometrial cancer remains controversial. Therefore, we quantitatively summarized the evidence from observational studies with a meta-analysis. Methods. We searched PubMed, Web of Science, Embase, Medline, Chinese National Knowledge Infrastructure (CNKI), and Wan Fang Med online up to March 2019, and all eligible casecontrol and cohort studies were included in the study. Pooled relative risks (RRs) with 95\% confidence intervals (CIs) were calculated using the random-effects model. The dose-response relationship was assessed by restricted cubic spline model. The heterogeneity among studies was evaluated by $\mathrm{I}^{2}$. Metaregression was used to explore the potential sources of between-study heterogeneity. Egger's test was used to estimate publication bias. Results. Eighteen articles including 957242 subjects with 4781 cases were included in the meta-analysis. The pooled RR (95\%CI) of endometrial cancer for the highest versus the lowest age at menopause was 1.89 (95\%CI: 1.58-2.26). For dose-response analysis, a nonlinear relationship was found between age at menopause and endometrial cancer, and the positive association became statistically significant when age at menopause was greater than 46.5 years old. Conclusions. This meta-analysis suggested that age at menopause was positively associated with endometrial cancer. For women whose menopausal age over 46.5 years old, the risk of endometrial cancer increased with the age at menopause.

\section{Introduction}

Endometrial cancer is the most common gynecological tumor of the female [1]. Globally, endometrial cancer causes approximately $5 \%$ of cancer cases and over $2 \%$ of cancer deaths in women $[2,3]$. It ranks the fourth most common malignant tumor in the female in developed countries [4]. Present studies indicate that genetic factors, anthropometric factors, lifestyle factors (e.g., tobacco smoking, alcohol drinking, physical activity, and usual diet), and clinically relevant diseases (e.g., diabetes, polycystic ovary) are related to endometrial cancer risk [5-14]. Besides, many reproductive factors that increase continuous stimulation of estrogen can also result in a higher risk of endometrial cancer, such as parity [15], age at menarche [16], oral contraceptive use [17], and breastfeeding [18].

Menopause as the terminus of women reproductive life is generally defined as a stop of menstruation for a consecutive year [19]. The age at menopause is of great clinical and public health significance [20]. Considering women with a later menopausal age have higher hormone levels and longer lifetime exposure to estrogens [21], age at menopause may be associated with many diseases. Studies had found that menopausal age was related to the risk of breast cancer and liver cancer [22-25]. However, the association between age at menopause and endometrial cancer is still controversial.

In order to explore the association between age at menopause and the risk of endometrial cancer, a large number of epidemiologic studies have been conducted [4, 2642]. Among these studies, ten studies suggested a significant association between later age at menopause and an increased risk of endometrial cancer $[4,26,30,32-36,38,41]$, but the effect size in different studies was various, whereas no significant association was found in the other eight studies $[27-29,31,37,39,40,42]$. Therefore, we conducted a metaanalysis to quantitatively evaluate the association between age at menopause and the risk of endometrial cancer risk. 

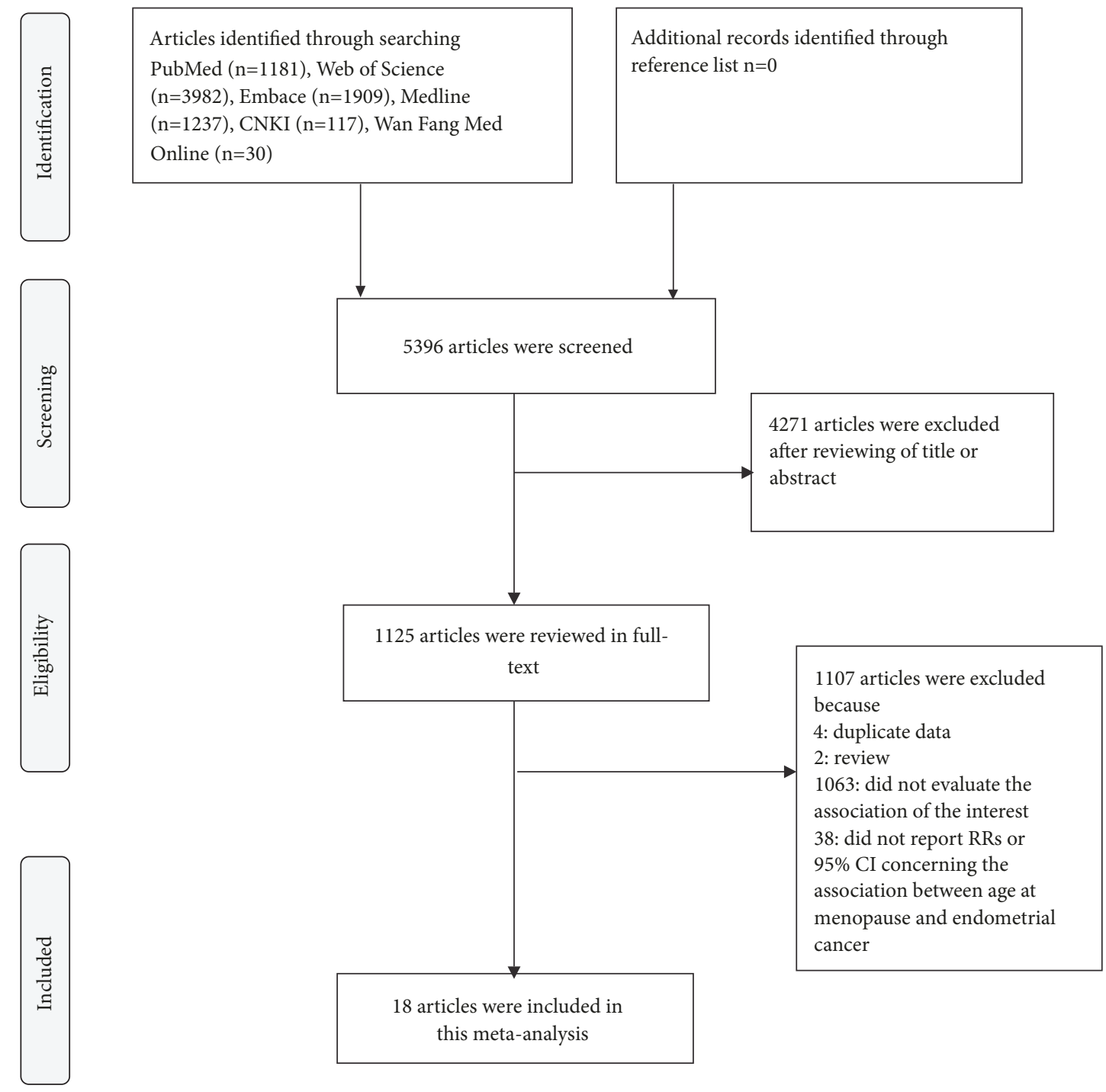

18 articles were included in this meta-analysis

FIGURE 1: Flow chart of the selection of studies included in the meta-analysis.

\section{Materials and Methods}

Preferred Reporting Items for Systematic reviews and MetaAnalyses (PRISMA) guidelines were adopted [43].

2.1. Search Strategy. We used extended computer-based searches to obtain available studies published in English or Chinese from the databases of PubMed, Web of Science, Embase, Medline, Chinese National Knowledge Infrastructure (CNKI), and Wan Fang Med Online. The search terms used to search articles in this study were "age at menopause" (or "menopaus* age" or "the age of postmenopause" or "age at climacteric" or "climacteric age" or "pausimenia age") and "endometrial cancer" (or "endometrial neoplasm" or "endometrial carcinoma" or "carcinoma of endometrium"). Relevant references within included studies were also manually searched. The detailed steps of the literature selection were shown in Figure 1.
2.2. Inclusion Criteria. If the article met the following characteristics, it would be included in our meta-analysis. (1) An observational study (cohort or case-control) was published as an original article. (2) The exposure of interest was categorized age at menopause. (3) The outcome of interest was endometrial cancer. (4) There was reported effect size (relative risk (RR) or odds ratio (OR) or hazard ratio (HR) or incidence rate ratio (IRR)) and 95\% confidence interval (CI) for the association between age at menopause and endometrial cancer. (5) We selected the most recent study if data from the same population were used in multiple articles.

All identified studies were searched and reviewed carefully by two investigators (Yanjun Wu and Wenjun Sun). If the two investigators had different views on the same article, it would be settled by discussing with the third investigator (Dongfeng Zhang). 


\section{Data Abstraction}

From each eligible article, we extracted the first author's name, country in which the study was performed, publication year, the type of study design, the follow-up duration of cohort study, age range or mean age at baseline, the number of cases, and controls in case-control studies as well as the person-year of cases in cohort studies. We also abstracted the information about age at menopause, RRs (we presented all results as RR for simplicity) with their 95\%CIs for each category of age at menopause, and adjustment factors in each study, menopausal type, the definition of postmenopausal status, and the source of case information.

\section{Quality Assessment}

The Newcastle-Ottawa Scale (NOS) [44] was used to assess the quality of case-control studies and cohort studies included in this study. The scale was composed of three parts (selection, comparability, and outcome), with a maximum score of 9 stars.

4.1. Statistical Analysis. The pooled measure was calculated as the inverse variance-weighted mean of the natural logarithm of RR with corresponding 95\% CI to assess the strength of association between age at menopause and the risk of endometrial cancer. Heterogeneity among studies was assessed by $I^{2}$ proposed by Higgins and Thompson [45]. Metaregression was performed to explore potential sources of between-study heterogeneity [46]. The influence analysis with one study removed at a time was carried out to evaluate whether a single study could affect the results significantly. Publication bias was evaluated using Egger's test and funnel plot [47].

For dose-response analysis, a two-stage random-effects dose-response meta-analysis [48] was performed. In the first stage, a restricted cubic spline model with three knots at the 25th, 50th, and 75th centiles of the levels of age at menopause was estimated using generalized least square regression [49], taking into account the correlation within each set of published RRs [50]. Then the study-specific estimates were combined using the restricted maximum likelihood method in a multivariate random-effects metaanalysis [51]. A $p$ value for nonlinearity was calculated by testing the null hypothesis that the coefficient of the second spline is equal to 0 . The details of the statistical method have been described elsewhere [52].

All statistical analyses were performed with StataV.15.0 (Stata Corp., College Station, TX, USA). All reported probabilities ( $P$ values) were two-sided, and $P$ values less than 0.05 were considered statistically significant.

\section{Results}

5.1. Study Selection. According to the search terms mentioned in the section of Materials and methods, we identified 1181 articles from PubMed, 3982 articles from Web of Science, 1909 articles from Embase, 1237 articles from Medline, 117 articles from CNKI, and 30 articles from Wan Fang Med Online. We excluded 4271 articles by reviewing the title and abstract. In the step of full-text article reviewing, we further excluded 1107 articles. Among them, four articles had the same population, two articles were reviews, 1063 articles failed to evaluate the association between menopausal age and endometrial cancer, and 38 articles did not have RRs (95\% CIs) concerning the interests. Ultimately, 18 articles $[4,26-42]$ were included in this meta-analysis. The detailed steps of the literature selection were presented in Figure 1.

5.2. Quality Assessment. After using NOS to assess the quality of the 18 articles included in this study, the mean NewcastleOttawa score was 7.8 (range from 6 to 9) for case-control studies and 8.1 (range from 7 to 9) for cohort studies. The detailed results of the quality assessment were summarized in Tables S1 and S2.

5.3. Study Characteristics. In the 18 articles, nine articles were case-control studies [27-29, 31-33, 36, 38, 41] and nine articles were cohort studies [4, 26, 30, 34, 35, 37, 39, 40, 42]. With regard to continent where the study was conducted, five studies were conducted in Asian [27, 29, 32, 34, 40], four studies $[4,26,36,41]$ in Europe, and nine studies in North America. The endometrial cancer cases of most studies [4, $26,27,30-33,35,38-42$ ] were identified from registry records (such as cancer registry) and five studies [28, 29, 34, 36, 37] were from hospital medical records. Six studies [27, 28, 32, $35,37,39$ ] included women only with natural menopause, five studies [31, 33, 34, 41, 42] included women with both natural menopause and surgical menopause, and other seven studies $[4,26,29,30,36,38,40]$ did not have relevant information. Information about the definition of postmenopausal status and the detailed characteristics of the included studies were shown in Table 1.

5.4. Association between Age at Menopause and Endometrial Cancer. The association between age at menopause and endometrial cancer was evaluated in 18 articles [4, 26-42] with 957242 participants and 4781 cases. We could observe a statistically positive association between age at menopause and the risk of endometrial cancer in 10 articles [4, 26, 30, 32$36,38,41]$ of them, whereas the other eight studies [27-29, $31,37,39,40,42]$ showed no obvious association. The pooled $\mathrm{RR}$ of the risk of endometrial cancer for the highest versus the lowest age at menopause was 1.89 (95\%CI 1.58-2.26; $I^{2}=$ $45.0 \%, P_{\text {for heterogeneity }}=0.021$ ) (Figure 2 ).

In the dose-response analysis of 15 articles [4, 27$34,36-38,40-42]$, a nonlinear association between age at menopause and endometrial cancer was found $\left(P_{\text {nonlinearity }}<\right.$ 0.01). The positive association became significant when age at menopause was greater than 46.5 years old. The RRs $(95 \%$ CIs) of endometrial cancer risk were 1.04 (1.03-1.06), 1.17 (1.14-1.20), 1.57 (1.45-1.71), and 2.08 (1.80-2.39) for 47, 50, 54, and 57 years old of age at menopause, respectively (Figure 3 ).

5.5. Subgroup Analysis. In the subgroup analysis by continent where the study was conducted, the pooled RRs 


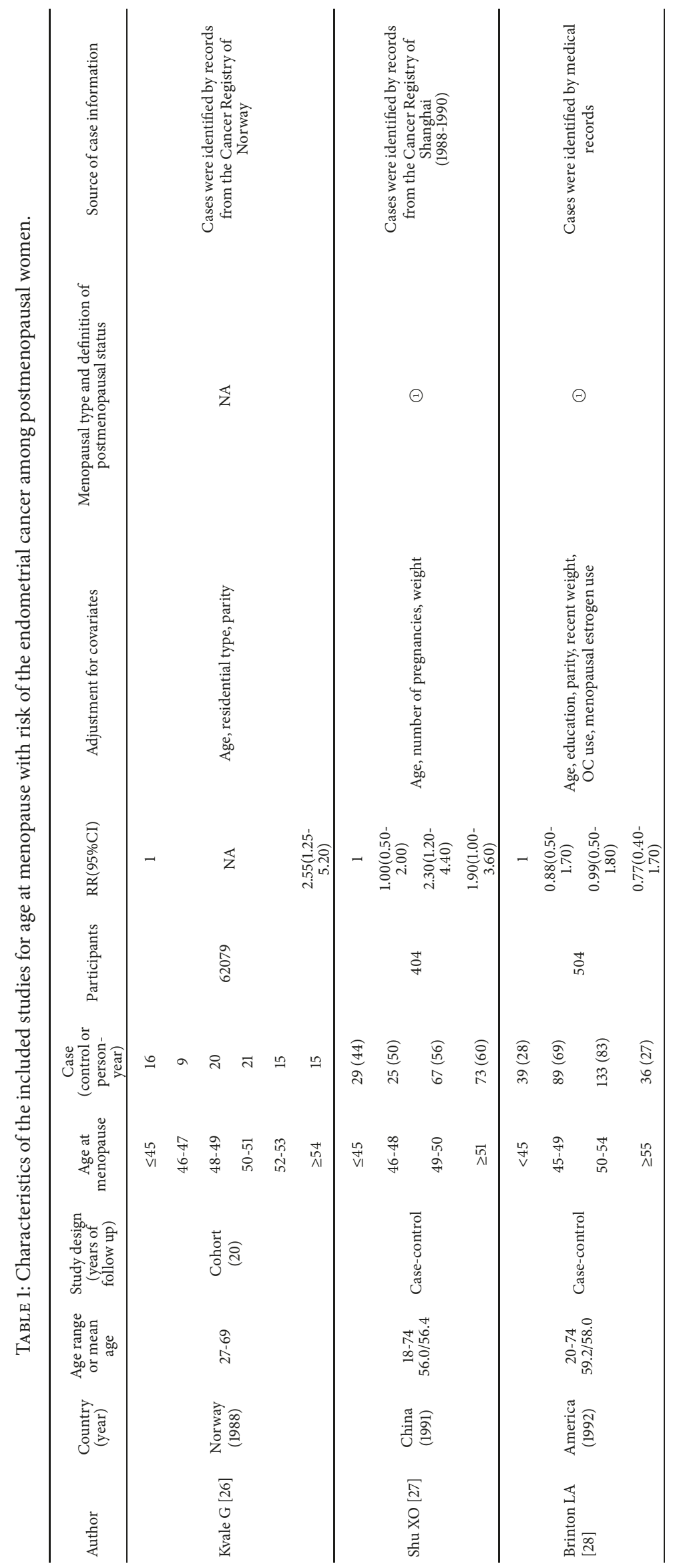




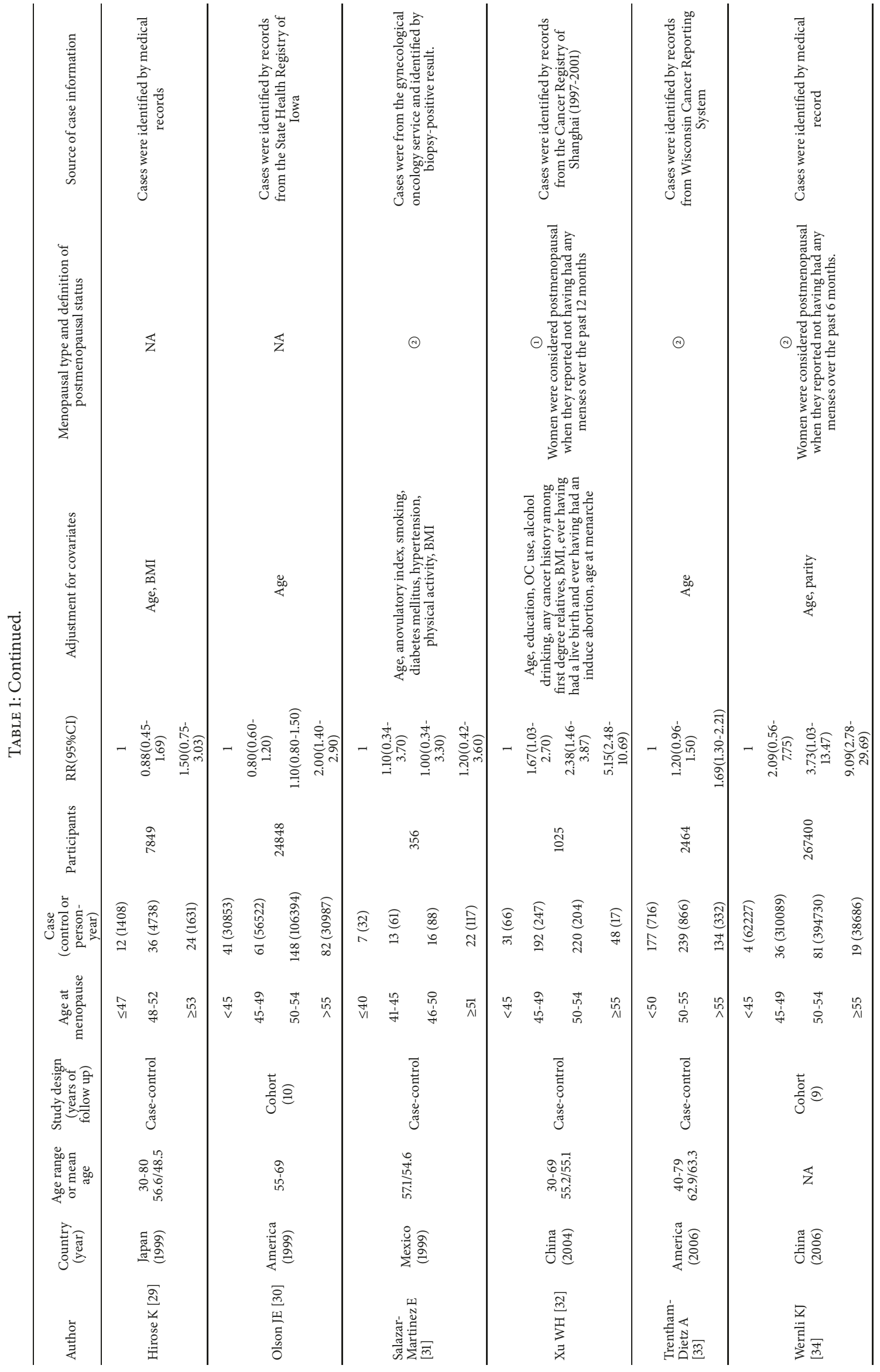




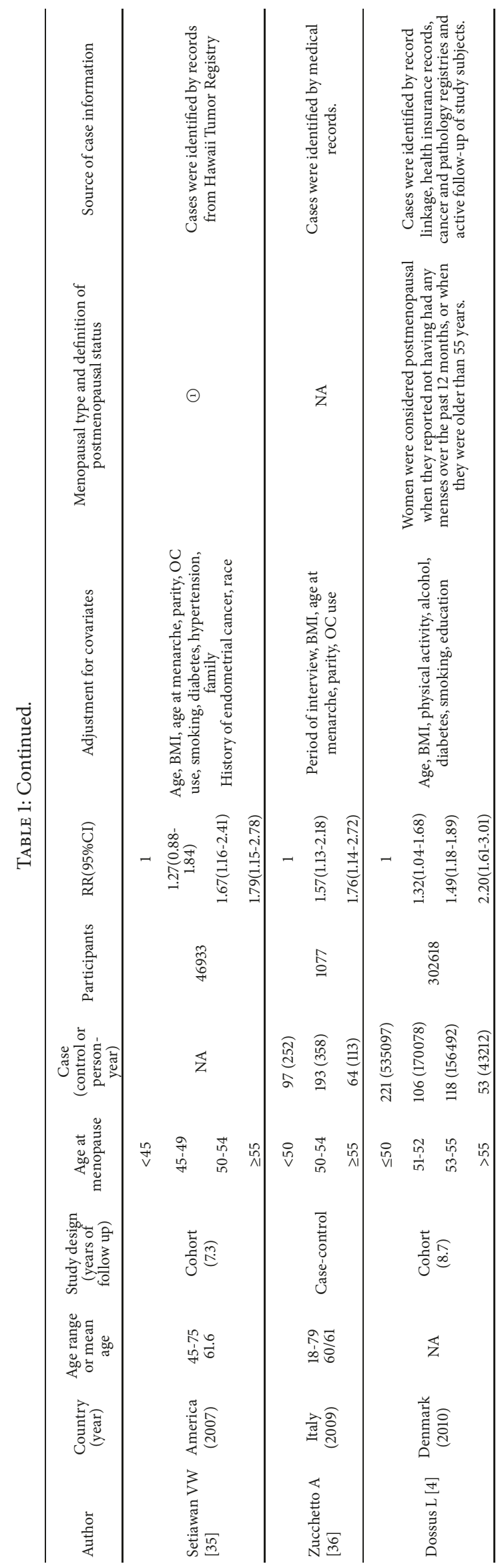




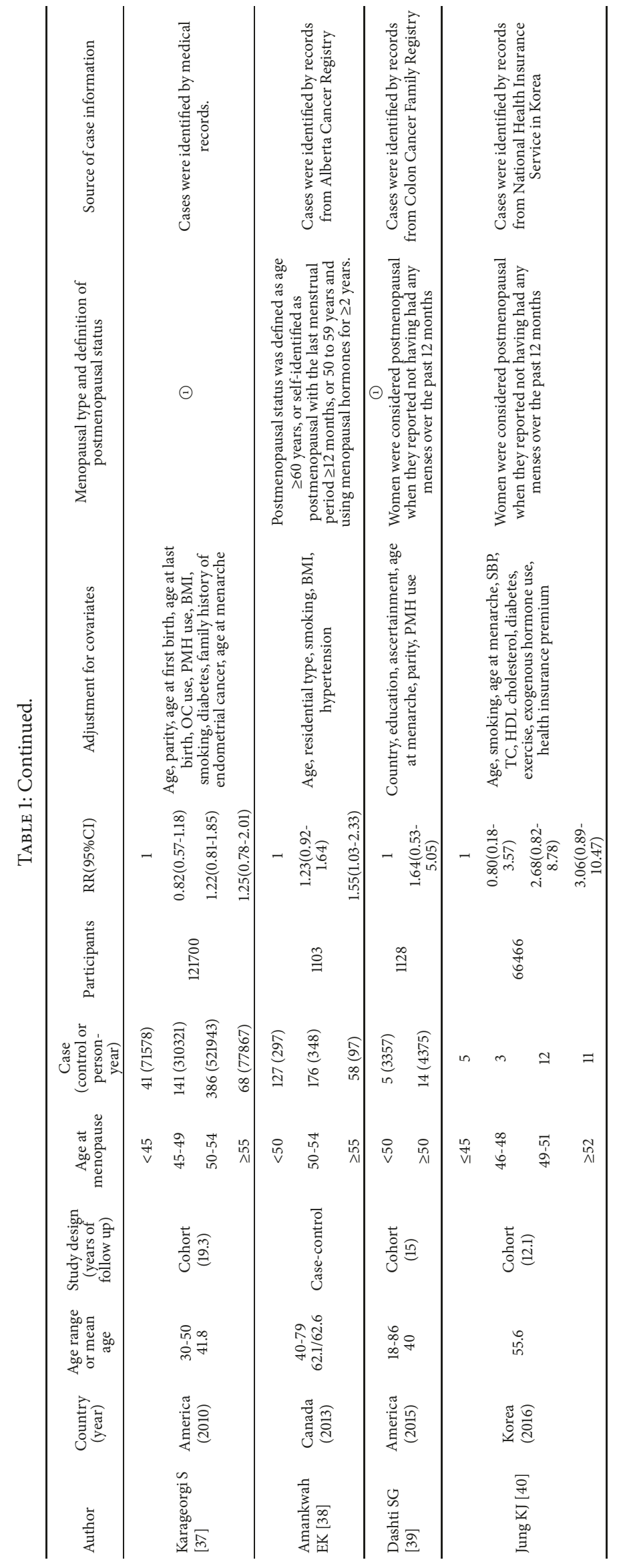




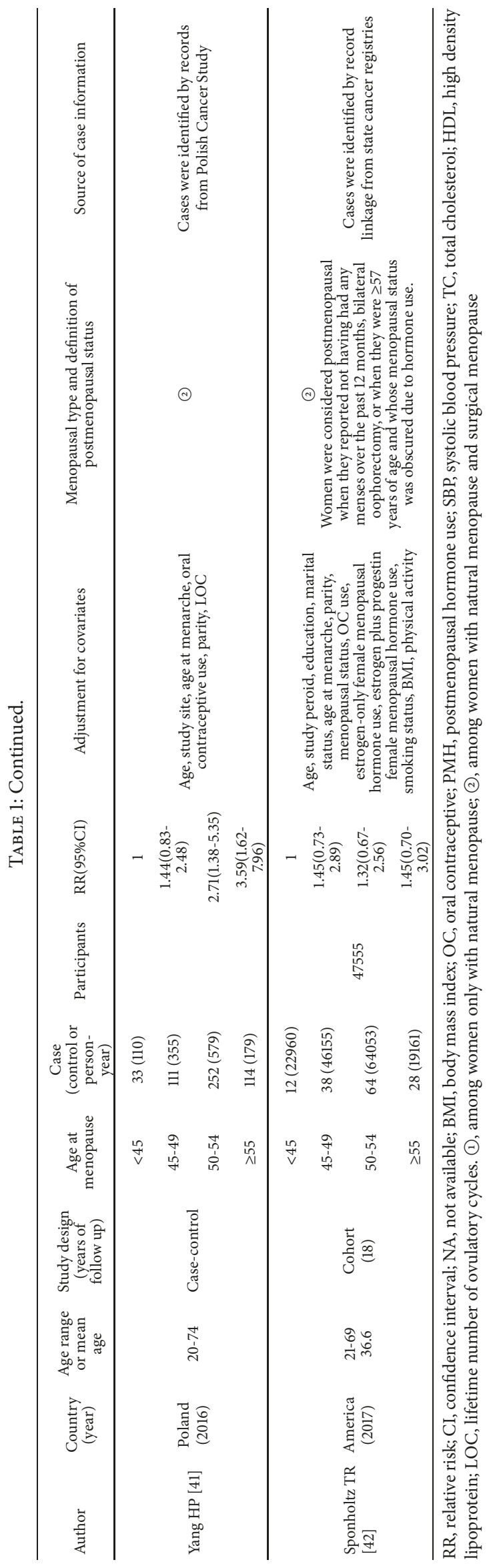




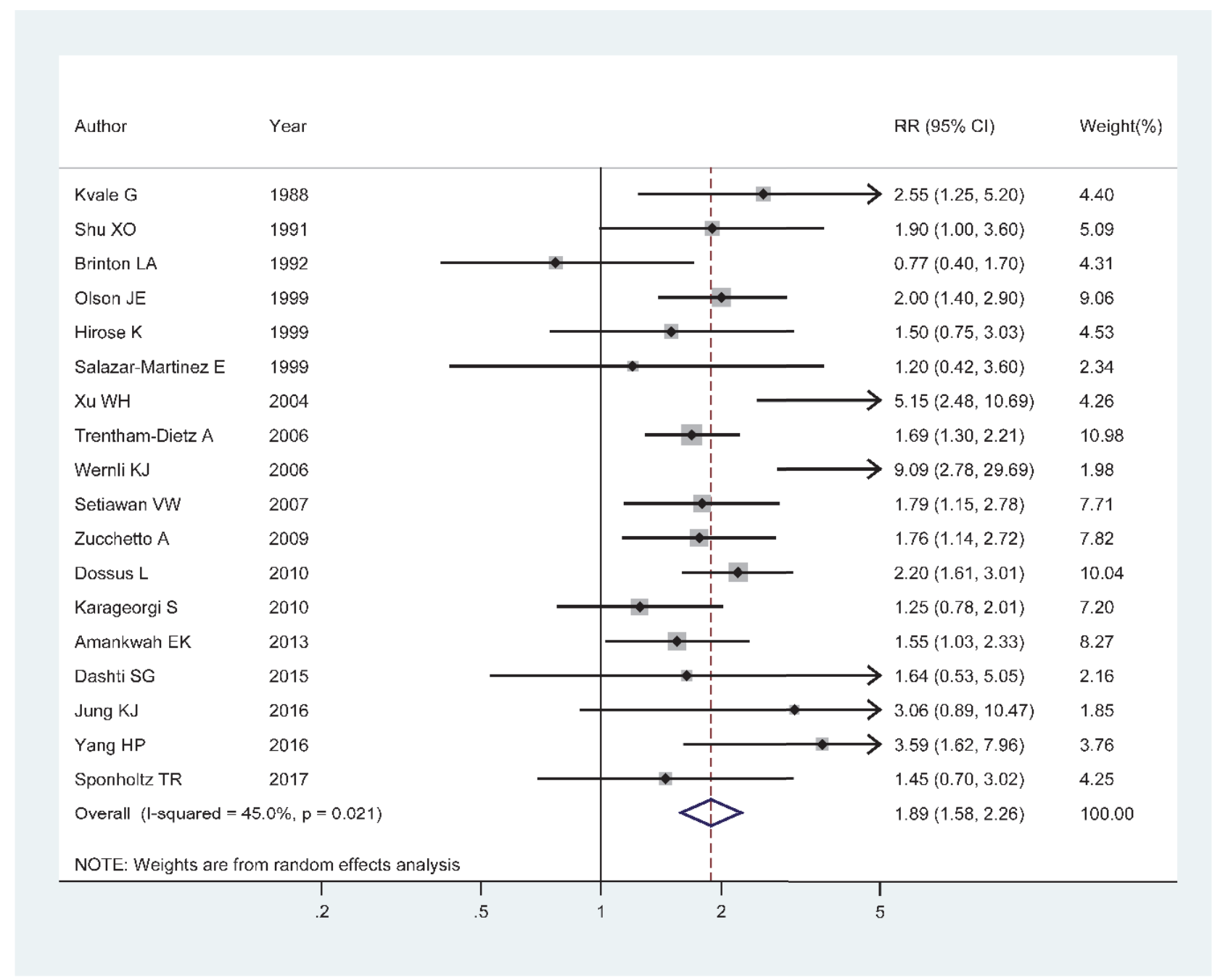

Figure 2: Forest plot of age at menopause and the risk of endometrial cancer. The size of gray box is positively proportional to the weight assigned to each study, and horizontal lines represent the $95 \%$ confidence intervals.

(95\%CIs) were $1.60(1.37-1.86), 3.06$ (1.64-5.72), and 2.19 (1.74-2.75) for North America, Asia, and Europe, respectively. In the subgroup analysis by study design, the pooled RRs (95\%CIs) for case-control studies and cohort studies were 1.80 (1.36-2.38) and 1.98 (1.56-2.52), separately. In the subgroup analysis performed according to menopausal type, the pooled RRs (95\%CIs) were 1.72 (1.09-2.71), 2.25 (1.31-3.85), and 1.94 (1.63-2.30) for studies among natural menopausal women, studies among both natural menopause and surgical menopause women, and studies without relevant information, respectively. In the subgroup analysis by reference group of menopausal age, the pooled RR (95\%CI) was 2.06 (1.51$2.81)$ for studies that used age at menopause $\leq 45(<45$ or $\leq 45$ or $\leq 40)$ as reference group and the pooled RR $(95 \% \mathrm{CI})$ was 1.79 (1.52-2.10) for studies that used age at menopause $\leq 50$ $(<50$ or $\leq 50$ or $\leq 47)$ as the reference group. In the subgroup by the Newcastle-Ottawa score, the pooled RRs (95\%CIs) were $1.67(1.12-2.49)$ and $1.98(1.66-2.37)$ for studies with a score of 6 or 7 and studies with a score of 8 or 9. The detailed results of subgroup analysis were summarized in Table 2.

5.6. Meta-Regression. Multivariable metaregression with the covariates of publication year $(p=0.727)$, study design $(p=0.623)$, mean age of study participants $(p=0.538)$, menopausal type $(p=0.621)$, reference group of menopausal age $(p=0.554)$, and the Newcastle-Ottawa score $(p=0.362)$ showed that these covariates had no significant impact on the heterogeneity. But continent where the study was conducted ( $p=0.014$ ) was found to have an influence on the betweenstudy heterogeneity.

5.7. Influence Analysis and Publication Bias. No study had excessive influence on the pooled RR for the highest versus the lowest age at menopause in the influence analysis. No evidence of significant small-study effect for the analyses was 


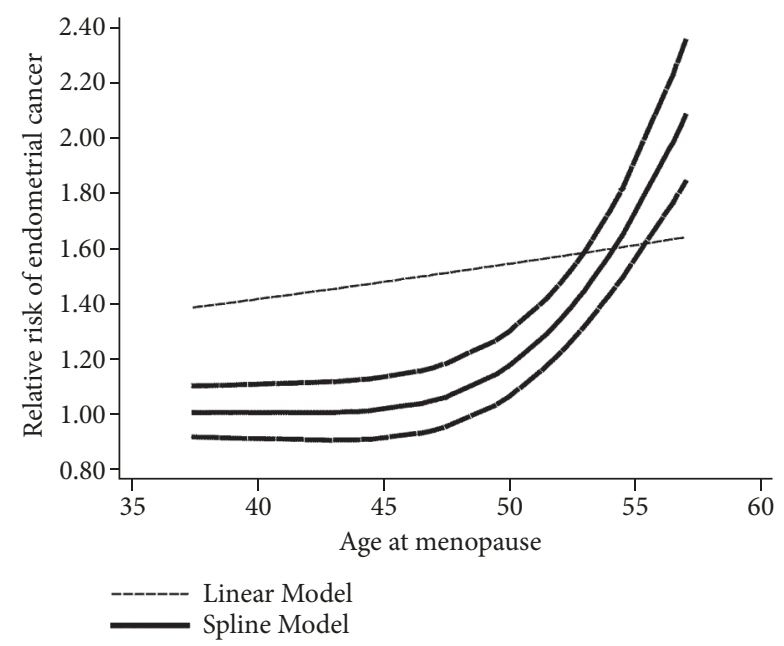

FIGURE 3: The dose-response analysis between age at menopause and the risk of endometrial cancer with restricted cubic splines in a multivariate random-effects dose-response model. The solid line and the long dash line represent the estimated relative risks and its 95\% CIs. Short dash line represents the linear relationship.

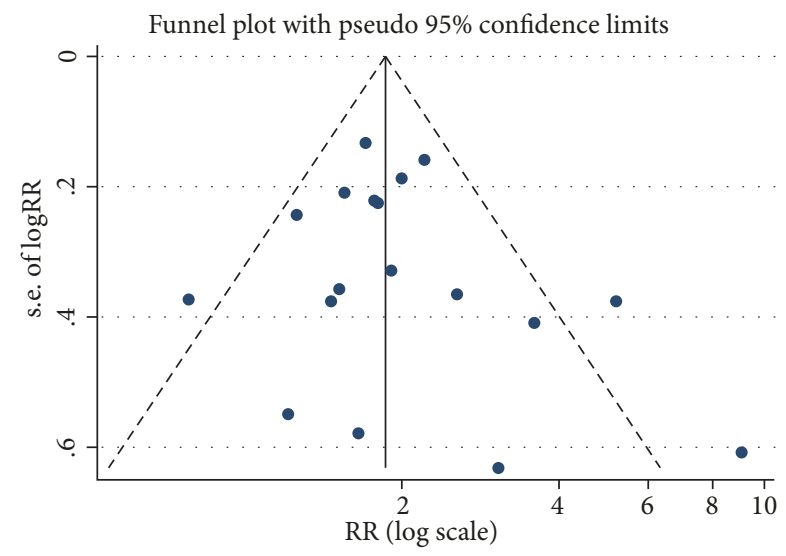

Figure 4: Funnel plot with pseudo 95\% confidence limits for the analysis of age at menopause and risk of endometrial cancer (RR, relative risk).

found by the visual inspection of the funnel plot and Egger's test $(p=0.373)$. The results of the funnel plot were shown in Figure 4 .

\section{Discussion}

The average menopausal age for women varied among region [53]. Studies also showed that overweight, later age at menarche, and higher parity were associated with later menopausal age $[54,55]$. In our study, we found that a later menopausal age was associated with an increased risk of endometrial cancer. In dose-analysis, a nonlinear relationship was found between age at menopause and endometrial cancer, and the positive association became statistically significant when age at menopause was greater than 46.5 years old. The positive association remained in subgroup analysis by continent, menopausal type, the reference group of menopausal age, and Newcastle-Ottawa score. Subgroup analysis especially by study design showed the positive association between age at menopause and endometrial cancer for cohort studies.

The mechanisms of higher endometrial cancer risk caused by later age at menopause are still equivocal. The hypothesis of "estrogen unopposed by progesterone" has been proposed as an important etiological factor $[56,57]$, which suggests that high levels of biological estrogens not counterbalanced by progesterone can result in a higher risk of endometrial cancer through increasing the mitotic activity of endometrial cells. Because the high-level female hormone can increase the probability of DNA-damaging (such as mitotic activity, DNA replication, and somatic mutations) and then become a fixed mutation [58, 59], people with a later menopausal age have higher hormone levels and longer time exposure to estrogens before menopause [21, 56]. In addition, later menopause might increase the risk of endometrial cancer by increasing the rate of spontaneous and environmentally induced mutations in endometrial stem cells [58]. Moreover, progesterone deficiency associated with anovulatory cycles is common in people having a later menopausal age, which may also contribute to endometrial cancer risk [60].

The issue of between-study heterogeneity should be paid particular attention in meta-analyses [61], and investigating the potential sources of between-study heterogeneity is necessary. The result of this meta-analysis showed moderate between-study heterogeneity in the analyses of age at menopause and risk of endometrial cancer. Multivariable metaregression with covariates of publication year, the continent where the study was conducted, study design, the mean age of study participants, menopausal type, reference group of menopausal age, and the Newcastle-Ottawa score was carried out to explore the potentially important source of heterogeneity. Among these covariates, just continent where the study was conducted was found to have a contribution to the between-study heterogeneity. After the subgroup analysis by continent, the positive association still remained in North America, Asia, and Europe and the pooled RRs were 1.60 $\left(95 \%\right.$ CI $\left.1.37-1.86 ; I^{2}=0.0 \% P_{\text {for heterogeneity }}=0.516\right), 3.06$ (95\% CI 1.64-5.72; $I^{2}=63.5 \% P_{\text {for heterogeneity }}=0.027$ ), and $2.19\left(95 \%\right.$ CI $\left.1.74-2.75 ; I^{2}=0.0 \% P_{\text {for heterogeneity }}=0.453\right)$, respectively.

The study has many virtues. First, compared with the original individual study, our meta-analysis has a large number of included participants, which can make the results more precise and more reliable. Second, the quality of the included articles was relatively high with NewcastleOttawa score ranging from 6 to 9. Third, the results are still meaningful after subgroup analysis by continent, menopausal type, the reference group of menopausal age, and NewcastleOttawa score, and subgroup analysis by study design also showed the positive association between age at menopause and endometrial cancer in cohort studies. Finally, we conducted a dose-response analysis to explore the association between age at menopause and endometrial cancer quantitatively. 
TABLE 2: Summary of relative risks (RRs) for association of age at menopause with risk of the endometrial cancer.

\begin{tabular}{|c|c|c|c|c|}
\hline Stratification & Number of studies & RR (95\% CI) & $I^{2}, \%$ & $P_{\text {for heterogeneity }}$ \\
\hline All studies & 18 & $1.89(1.58-2.26)$ & $45.0 \%$ & 0.021 \\
\hline \multicolumn{5}{|l|}{ Continent } \\
\hline North America & 9 & $1.60(1.37-1.86)$ & $0.0 \%$ & 0.516 \\
\hline Asia & 5 & $3.06(1.64-5.72)$ & $63.5 \%$ & 0.027 \\
\hline Europe & 4 & $2.19(1.74-2.75)$ & $0.0 \%$ & 0.453 \\
\hline \multicolumn{5}{|l|}{ Study Design } \\
\hline Case-control study & 9 & $1.80(1.36-2.38)$ & $54.5 \%$ & 0.024 \\
\hline Cohort study & 9 & $1.98(1.56-2.52)$ & $35.4 \%$ & 0.135 \\
\hline \multicolumn{5}{|l|}{ Menopausal type } \\
\hline Natural menopause & 6 & $1.72(1.09-2.71)$ & $67.1 \%$ & 0.009 \\
\hline Natural menopause and surgical menopause & 5 & $2.25(1.31-3.85)$ & $64.0 \%$ & 0.025 \\
\hline NA & 7 & $1.94(1.63-2.30)$ & $0.0 \%$ & 0.729 \\
\hline \multicolumn{5}{|l|}{ Reference group of menopausal age } \\
\hline$\leq 45(<45$ or $\leq 45$ or $\leq 40)$ & 12 & $2.06(1.51-2.81)$ & $60.6 \%$ & 0.003 \\
\hline$\leq 50(<50$ or $\leq 50$ or $\leq 47)$ & 6 & $1.79(1.52-2.10)$ & $0.0 \%$ & 0.761 \\
\hline \multicolumn{5}{|l|}{ The Newcastle-Ottawa score } \\
\hline 6 or 7 & 5 & $1.59(1.18-2.15)$ & $27.1 \%$ & 0.241 \\
\hline 8 or 9 & 13 & $2.05(1.64-2.56)$ & $50.8 \%$ & 0.018 \\
\hline
\end{tabular}

$\mathrm{RR}$, relative risk; CI, confidence interval; BMI, body mass index; $\mathrm{PMH}$, postmenopausal hormone

However, there are also several potential limitations in our study. First, the adjusted confounders are different among studies and the residual confounding could not be eliminated thoroughly. Second, the age range of participant is disparate in every study and years of follow-up in cohort studies are diverse. Finally, the definition of postmenopausal status and the stratification of age varied among studies, which might affect the result.

\section{Conclusions}

Results from this meta-analysis indicated that age at menopause was positively associated with the risk of endometrial cancer. When the menopausal age of women exceeded 46.5 years, the risk of endometrial cancer increased with her menopausal age. For these women, they should develop good lifestyles to reduce the risk of endometrial cancer. More well-designed prospective studies are needed to confirm the association in the future.

\section{Disclosure}

Ms Yanjun Wu and Dr Wenjun Sun are co-first authors.

\section{Conflicts of Interest}

We declare that we have no conflicts of financial and commercial interest.

\section{Acknowledgments}

Thanks are due to all the authors of included paper.

\section{Supplementary Materials}

Table S1. Quality assessment of included case-control studies. Table S2. Quality assessment of included cohort studies. (Supplementary Materials)

\section{References}

[1] M. M. Braun, E. A. Overbeek-Wager, and R. J. Grumbo, "Diagnosis and management of endometrial cancer," American Family Physician, vol. 93, no. 6, pp. 468-474, 2016.

[2] M. A. Clarke, B. J. Long, A. Del Mar Morillo, M. Arbyn, J. N. Bakkum-Gamez, and N. Wentzensen, "Association of endometrial cancer risk with postmenopausal bleeding in women: a systematic review and meta-analysis," JAMA Internal Medicine, vol. 178, no. 9, pp. 1210-1222, 2018.

[3] J. Ferlay, I. Soerjomataram, R. Dikshit et al., "Cancer incidence and mortality worldwide: sources, methods and major patterns in GLOBOCAN," International Journal of Cancer, vol. 136, pp. E359-E386, 2015.

[4] L. Dossus, N. Allen, R. Kaaks et al., "Reproductive risk factors and endometrial cancer: the european prospective investigation into cancer and nutrition," International Journal of Cancer, vol. 127, pp. 442-451, 2010.

[5] N. Pabalan, M. R. Pineda, H. Jarjanazi, D. M. Christofolini, C. P. Barbosa, and B. Bianco, "Association of the $+331 \mathrm{G} / \mathrm{A}$ progesterone receptor gene $(\mathrm{PgR})$ polymorphism with risk of endometrial cancer in caucasian women: a meta-analysis," Archives of Gynecology and Obstetrics, vol. 291, no. 1, pp. 115-122, 2015.

[6] B. Zhou, L. Yang, Q. Sun et al., "Cigarette smoking and the risk of endometrial cancer: a meta-analysis," American Journal of Medicine, vol. 121, no. 6, pp. 501.e3-508.e3, 2008.

[7] D. Schmid, G. Behrens, M. Keimling, C. Jochem, C. Ricci, and M. Leitzmann, "A systematic review and meta-analysis of 
physical activity and endometrial cancer risk," European Journal of Epidemiology, vol. 30, no. 5, pp. 397-412, 2015.

[8] C. Liao, D. Zhang, C. Mungo, D. Andrew Tompkins, and A. M. Zeidan, "Is diabetes mellitus associated with increased incidence and disease-specific mortality in endometrial cancer? a systematic review and meta-analysis of cohort studies," Gynecologic Oncology, vol. 135, no. 1, pp. 163-171, 2014.

[9] D. Aune, D. A. N. Rosenblatt, D. S. M. Chan et al., "Anthropometric factors and endometrial cancer risk: a systematic review and dose-response meta-analysis of prospective studies," Annals of Oncology: Official Journal of the European Society for Medical Oncology, vol. 26, no. 8, pp. 1635-1648, 2015.

[10] Q. Zhou, P. Guo, H. Li, and X.-D. Chen, "Does alcohol consumption modify the risk of endometrial cancer? a dose-response meta-analysis of prospective studies," Archives of Gynecology and Obstetrics, vol. 295, no. 2, pp. 467-479, 2017.

[11] C.-J. Si, L. Shu, P.-F. Zheng et al., "Dietary patterns and endometrial cancer: A meta-analysis," European Journal of Cancer Prevention: The Official Journal of the European Cancer Prevention Organisation (ECP), vol. 26, no. 4, pp. 336-345, 2017.

[12] A. K. Win, J. C. Reece, and S. Ryan, "Family history and risk of endometrial cancer : A systematic review and meta-analysis," Obstetrics \& Gynecology, vol. 125, no. 1, pp. 89-98, 2015.

[13] Z. Haoula, M. Salman, and W. Atiomo, "Evaluating the association between endometrial cancer and polycystic ovary syndrome," Human Reproduction, vol. 27, no. 5, pp. 1327-1331, 2012.

[14] X. Tao, A. Jiang, L. Yin, Y. Li, F. Tao, and H. Hu, "Body mass index and age at natural menopause: a meta-analysis," Menopause (New York, NY), vol. 22, no. 4, pp. 469-474, 2015.

[15] Q. Wu, Y. Y. Li, C. Tu et al., "Parity and endometrial cancer risk: a meta-analysis of epidemiological studies," Scientific Reports, vol. 5, Article ID 14243, 2015.

[16] T. T. Gong, Y. L. Wang, and X. X. Ma, "Age at menarche and endometrial cancer risk: a dose-response meta-analysis of prospective studies," Scientific Reports, vol. 5, no. 1, Article ID 14051, 2015.

[17] N. Allen, R. Peto, V. Beral et al., "Endometrial cancer and oral contraceptives: an individual participant meta-analysis of 27 276 women with endometrial cancer from 36 epidemiological studies," Lancet Oncology, vol. 16, pp. 1061-1070, 2015.

[18] B. Zhan, X. Liu, F. Li, and D. Zhang, "Breastfeeding and the incidence of endometrial cancer: a meta-analysis," Oncotarget, vol. 6, no. 35, pp. 38398-38409, 2015.

[19] M. Glisic, N. Kastrati, J. Musa et al., "Phytoestrogen supplementation and body composition in postmenopausal women: a systematic review and meta-analysis of randomized controlled trials," Maturitas, vol. 115, pp. 74-83, 2018.

[20] E. B. Gold, "The timing of the age at which natural menopause occurs," Obstetrics \& Gynecology Clinics of North America, vol. 38, no. 3, pp. 425-440, 2011.

[21] R. Kaaks, A. Lukanova, and M. S. Kurzer, "Obesity, endogenous hormones, and endometrial cancer risk: a synthetic review," Cancer Epidemiology, Biomarkers \& Prevention: A Publication of The American Association for Cancer Research, Cosponsored by the American Society of Preventive Oncology, vol. 11, pp. 1531$1543,2002$.

[22] Collaborative Group on Hormonal Factors in Breast Cancer, "Menarche, menopause, and breast cancer risk: individual participant meta-analysis, including 118964 women with breast cancer from 117 epidemiological studies," The Lancet Oncology, vol. 13, no. 11, pp. 1141-1151, 2012.
[23] G.-C. Zhong, Y. Liu, N. Chen et al., "Reproductive factors, menopausal hormone therapies and primary liver cancer risk: a systematic review and dose-response meta-analysis of observational studies," Human Reproduction Update, vol. 23, no. 1, pp. 126-138, 2016.

[24] M. Kanazir, I. Boricic, D. Delic et al., "Risk factors for hepatocellular carcinoma: a case-control study in belgrade (serbia)," Tumori, vol. 96, no. 6, pp. 911-917, 2010.

[25] L. A. Mucci, H. E. Kuper, R. Tamimi, P. Lagiou, E. Spanos, and D. Trichopoulos, "Age at menarche and age at menopause in relation to hepatocellular carcinoma in women," British Journal of Obstetrics and Gynaecology, vol. 108, no. 3, pp. 291-294, 2001.

[26] G. Kvale, I. Heuch, and G. Ursin, "Reproductive factors and risk of cancer of the uterine corpus: a prospective study," Cancer Research, vol. 48, no. 21, pp. 6217-6221, 1988.

[27] X. Shu, L. A. Brinton, W. Zheng, Y. T. Gao, J. Fan, and J. F. Fraumeni, "A population-based case-control study of endometrial cancer in shanghai, china," International Journal of Cancer, vol. 49, no. 1, pp. 38-43, 1991.

[28] L. A. Brinton, M. L. Berman, R. Mortel et al., "Reproductive, menstrual, and medical risk factors for endometrial cancer: results from a case-control study," American Journal of Obstetrics \& Gynecology, vol. 167, no. 5, pp. 1317-1325, 1992.

[29] K. Hirose, K. Tajima, N. Hamajima et al., "Comparative casereferent study of risk factors among hormone-related female cancers in Japan," Japanese Journal of Cancer Research, vol. 90, no. 3, pp. 255-261, 1999.

[30] J. E. Olson, T. A. Sellers, K. E. Anderson, and A. R. Folsom, "Does a family history of cancer increase the risk for postmenopausal endometrial carcinoma? a prospective cohort study and a nested case-control family study of older women," Cancer, vol. 85, no. 11, pp. 2444-2449, 1999.

[31] E. Salazar-Martinez, E. C. Lazcano-Ponce, G. G. Lira-Lira, P. Escudero-De Los Rios, J. Salmeron-Castro, and M. HernandezAvila, "Reproductive factors of ovarian and endometrial cancer risk in a high fertility population in Mexico," Cancer Research, vol. 59, no. 15, pp. 3658-3662, 1999.

[32] W.-H. Xu, Y.-B. Xiang, Z.-X. Ruan et al., "Menstrual and reproductive factors and endometrial cancer risk: results from a population-based case-control study in urban shanghai," International Journal of Cancer, vol. 108, no. 4, pp. 613-619, 2004.

[33] A. Trentham-Dietz, H. B. Nichols, J. M. Hampton, and P. A. Newcomb, "Weight change and risk of endometrial cancer," International Journal of Epidemiology, vol. 35, no. 1, pp. 151-158, 2006.

[34] K. J. Wernli, R. M. Ray, D. L. Gao, A. J. De Roos, H. Checkoway, and D. B. Thomas, "Menstrual and reproductive factors in relation to risk of endometrial cancer in Chinese women," Cancer Causes \& Control, vol. 17, no. 7, pp. 949-955, 2006.

[35] V. W. Setiawan, M. C. Pike, L. N. Kolonel, A. M. Nomura, M. T. Goodman, and B. E. Henderson, "Racial/ethnic differences in endometrial cancer risk: the multiethnic cohort study," American Journal of Epidemiology, vol. 165, no. 3, pp. 262-270, 2007.

[36] A. Zucchetto, D. Serraino, J. Polesel et al., "Hormone-related factors and gynecological conditions in relation to endometrial cancer risk," European Journal of Cancer Prevention, vol. 18, no. 4, pp. 316-321, 2009.

[37] S. Karageorgi, S. E. Hankinson, P. Kraft, and I. De Vivo, "Reproductive factors and postmenopausal hormone use in relation to endometrial cancer risk in the Nurses' Health Study 
cohort 1976-2004," International Journal of Cancer, vol. 126, no. 1, pp. 208-216, 2010.

[38] E. K. Amankwah, C. M. Friedenreich, A. M. Magliocco et al., "Hormonal and reproductive risk factors for sporadic microsatellite stable and unstable endometrial tumors," Cancer Epidemiology, Biomarkers \& Prevention, vol. 22, no. 7, pp. 13251331, 2013.

[39] S. G. Dashti, R. Chau, D. A. Ouakrim et al., "Female hormonal factors and the risk of endometrial cancer in Lynch syndrome," Journal of the American Medical Association, vol. 314, no. 1, pp. 61-71, 2015.

[40] K. J. Jung, C. Park, Y. D. Yun, and S. H. Jee, "Duration of ovarian hormone exposure and gynecological cancer risk in korean women: the korean heart study," Cancer Epidemiology, vol. 41, pp. 1-7, 2016.

[41] H. P. Yang, K. R. Murphy, R. M. Pfeiffer et al., "Lifetime number of ovulatory cycles and risks of ovarian and endometrial cancer among postmenopausal women," American Journal of Epidemiology, vol. 183, no. 9, pp. 800-814, 2016.

[42] T. R. Sponholtz, J. R. Palmer, L. Rosenberg, E. E. Hatch, L. L. Adams-Campbell, and L. A. Wise, "Reproductive factors and incidence of endometrial cancer in U.S. black women," Cancer Causes \& Control, vol. 28, no. 6, pp. 579-588, 2017.

[43] D. Moher, A. Liberati, J. Tetzlaff, and D. G. Altman, "Preferred reporting items for systematic reviews and meta-analyses: the PRISMA statement," International Journal of Surgery, vol. 8, no. 5, pp. 336-341, 2010.

[44] G. Wells, B. Shea, D. O'Connell et al., “The newcastle-ottawa scale (NOS) for assessing the quality of nonrandomised studies in metaanalyses," 2016, http://www.ohri.ca/programs/clinical_ epidemiology/oxford.asp.

[45] J. P. T. Higgins and S. G. Thompson, "Quantifying heterogeneity in a meta-analysis," Statistics in Medicine, vol. 21, no. 11, pp. 15391558, 2002.

[46] J. P. T. Higgins and S. G. Thompson, "Controlling the risk of spurious findings from meta-regression," Statistics in Medicine, vol. 23, no. 11, pp. 1663-1682, 2004.

[47] M. Egger, G. D. Smith, M. Schneider, and C. Minder, "Bias in meta-analysis detected by a simple, graphical test," British Medical Journal (Clinical research ed), vol. 315, pp. 629-634, 1997.

[48] N. Orsini, R. Li, A. Wolk, P. Khudyakov, and D. Spiegelman, "Meta-analysis for linear and nonlinear dose-response relations: examples, an evaluation of approximations, and software," American Journal of Epidemiology, vol. 175, no. 1, pp. 66-73, 2012.

[49] F. E. Harre Jr., K. L. Lee, and B. G. Pollock, "Regression models in clinical studies: determining relationships between predictors and response," Journal of the National Cancer Institute, vol. 80, no. 15, pp. 1198-1202, 1988.

[50] N. Orsini, R. Bellocco, and S. Greenland, "Generalized least squares for trend estimation of summarized dose-response data," Stata Journal, vol. 6, no. 1, pp. 40-57, 2006.

[51] D. Jackson, I. R. White, and S. G. Thompson, "Extending DerSimonian and Laird's methodology to perform multivariate random effects meta-analyses," Statistics in Medicine, vol. 29, no. 12, pp. 1282-1297, 2010.

[52] J. Wang, J. Lv, W. Wang, and X. Jiang, "Alcohol consumption and risk of periodontitis: a meta-analysis," Journal of Clinical Periodontology, vol. 43, no. 7, pp. 572-583, 2016.
[53] S. Palacios, V. W. Henderson, N. Siseles, D. Tan, and P. Villaseca, "Age of menopause and impact of climacteric symptoms by geographical region," Climacteric: The Journal of The International Menopause Society, vol. 13, no. 5, pp. 419-428, 2010.

[54] F. Parazzini, "Determinants of age at menopause in women attending menopause clinics in Italy," Maturitas, vol. 56, no. 3, pp. 280-287, 2007.

[55] D. Zhu, H.-F. Chung, N. Pandeya et al., "Body mass index and age at natural menopause: an international pooled analysis of 11 prospective studies," European Journal of Epidemiology, vol. 33, no. 8, pp. 699-710, 2018.

[56] B. Pettersson, H. Adami, R. Bergström, and E. D. Johansson, "Menstruation span-a time-limited risk factor for endometrial carcinoma," Acta Obstetricia et Gynecologica Scandinavica, vol. 65, no. 3, pp. 247-255, 1986.

[57] T. J. A. Key and M. C. Pike, "The dose-effect relationship between "unopposed" oestrogens and endometrial mitotic rate: its central role in explaining and predicting endometrial cancer risk," British Journal of Cancer, vol. 57, no. 2, pp. 205-212, 1988.

[58] M. C. Pike, C. L. Pearce, and A. H. Wu, "Prevention of cancers of the breast, endometrium and ovary," Oncogene, vol. 23, no. 38, pp. 6379-6391, 2004.

[59] A. Akhmedkhanov, A. Zeleniuch-Jacquotte, and P. Toniolo, "Role of exogenous and endogenous hormones in endometrial cancer: review of the evidence and research perspectives," Annals of the New York Academy of Sciences, vol. 943, pp. 296315, 2001.

[60] J. M. Elwood, P. Cole, K. J. Rothman, and S. D. Kaplan, "Epidemiology of endometrial cancer," Journal of the National Cancer Institute, vol. 59, no. 4, pp. 1055-1060, 1977.

[61] M. R. Munafò and J. Flint, "Meta-analysis of genetic association studies," Trends in Genetics : TIG, vol. 20, no. 9, pp. 439-444, 2004. 


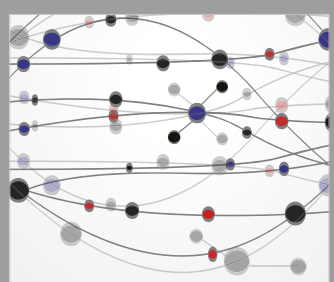

The Scientific World Journal
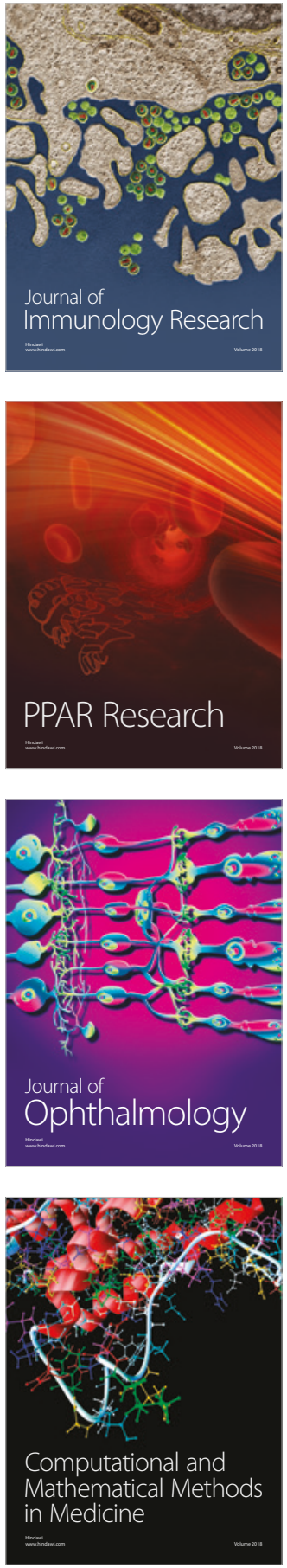

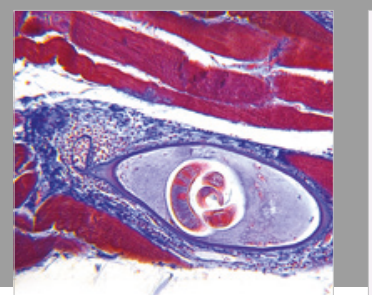

Gastroenterology Research and Practice

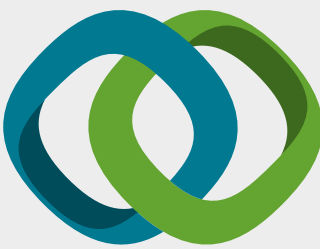

\section{Hindawi}

Submit your manuscripts at

www.hindawi.com
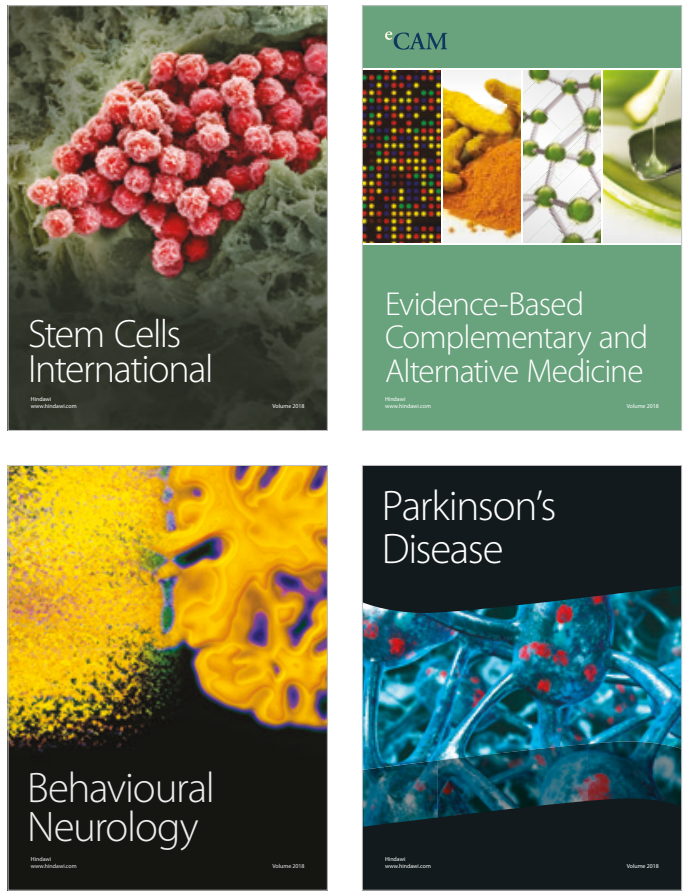

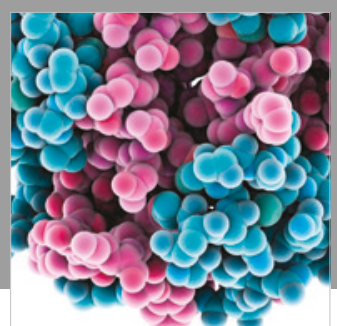

ournal of

Diabetes Research

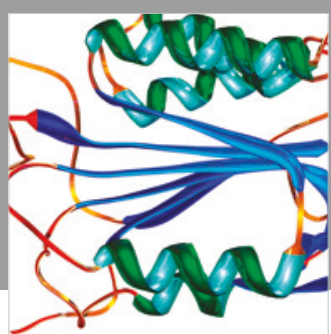

Disease Markers
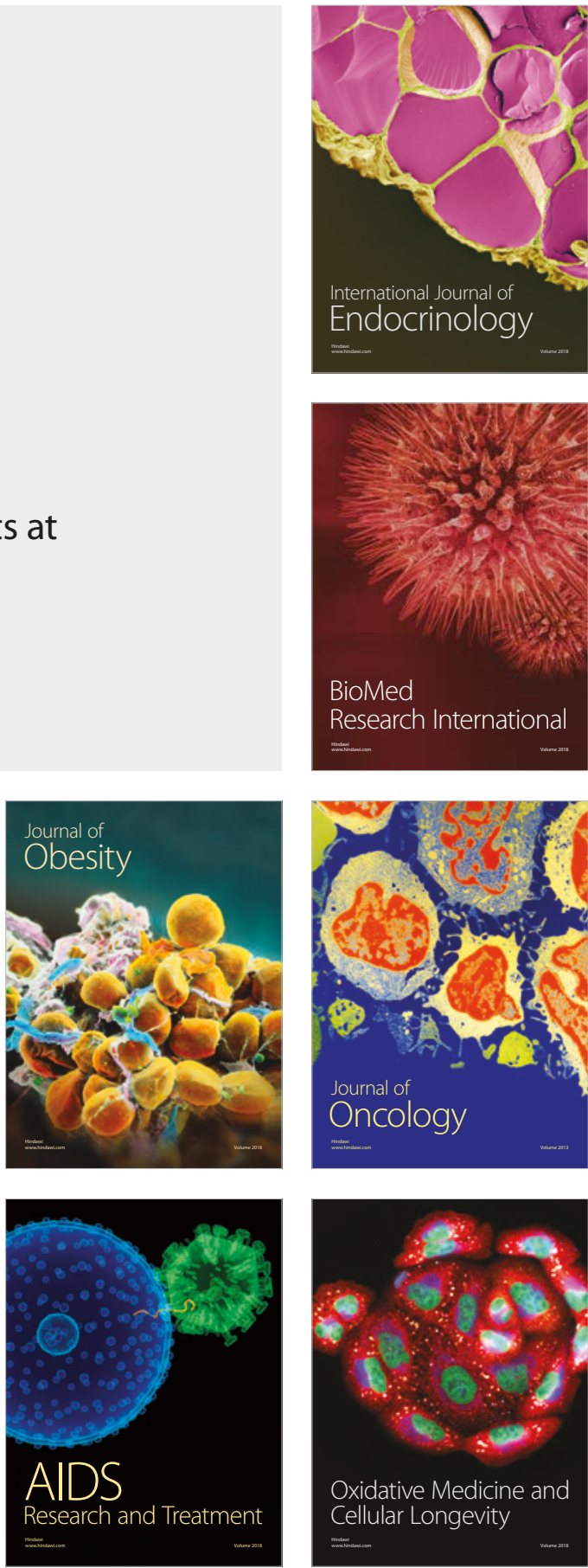